\title{
Réécriture des mythes littéraires dans le théâtre de Lina Prosa
}

Francesco D'Antonio

\section{(2) OpenEdition}

1 Journals

Édition électronique

URL : https://journals.openedition.org/cher/2059

DOI : 10.4000/cher.2059

ISSN : 2803-5992

Éditeur

Presses universitaires de Strasbourg

\section{Édition imprimée}

Date de publication : 29 mai 2018

Pagination : $51-60$

ISBN : 979-10-344-0019-5

ISSN : 1968-035X

\section{Référence électronique}

Francesco D'Antonio, «Réécriture des mythes littéraires dans le théâtre de Lina Prosa », reCHERches [En ligne], 20 | 2018, mis en ligne le 01 décembre 2021, consulté le 02 décembre 2021. URL : http:// journals.openedition.org/cher/2059; DOI : https://doi.org/10.4000/cher.2059

\section{(c) (i) (2)(2)}

Ce(tte) œuvre est mise à disposition selon les termes de la Licence Creative Commons Attribution Pas d'Utilisation Commerciale - Partage dans les Mêmes Conditions 4.0 International. 


\title{
Réécriture des mythes littéraires dans le théâtre de Lina Prosa
}

\author{
FRANCESCO D'ANTONIO
}

U ne large partie du théâtre de Lina Prosa est construite sur le principe de réécriture de mythes littéraires présents à différents niveaux, notamment dans les pièces Cassandre on the road (1998), Bang, Bang/in Care - Filottete et le rond infini (2004), Programme-Penthésilée (2008?) ainsi que la Trilogie du naufrage - Lampedusa beach (2003), Lampedusa snow (2012) - et Lampedusa way (2014). Dès les titres, ces pièces nous disent quelles mettent en scène ou évoquent des personnages mythologiques, très souvent des personnages féminins comme Cassandre ou Penthésilée. Des personnages qui font partie de la tradition épique et qui de cette dernière sont passés dans la tradition théâtrale d'abord gréco-latine et ensuite occidentale.

Cette circulation dans l'espace et dans le temps des récits et des personnages mythologiques évoque la question de la recréation ou de la réécriture des mythes au théâtre comme l'une des pratiques les plus répandues du théâtre antique, moderne et contemporain. Chez les auteurs dramatiques grecs cette réécriture consistait à prélever des fragments mythiques de la tradition épique pour les utiliser dans une nouvelle composition d'événements qui constituait une intrigue tragique capable de dire les universels. Peu importe si ces événements n'étaient pas réels. Au xx ${ }^{\mathrm{e}}$ siècle, le théâtre revient vers la pensée tragique antique et récupère la fonction mythique du théâtre pour briser l'ordre mimétique de la représentation (Sacco 2012: 9-15).

Dans le théâtre italien, la production dramaturgique de Lina Prosa, comme déjà celle de Pier Paolo Pasolini, s'insère dans cette pratique de la recréation, de la réécriture du mythe. Mais que signifie aujourd'hui réécrire les mythes au théâtre et quels sont les objectifs de cette écriture dans le théâtre de Lina Prosa?

1 Maître de conférences à l'Université de Strasbourg. 


\section{Une auteure dramatique entre réalité, mythe et science}

Née en Sicile, à Calatafimi, petit village près de Segesta, site archéologique grec qui a profondément influencé sa formation, Lina Prosa est auteure dramatique, metteure en scène, journaliste et poète. Elle vit et travaille à Palerme. Depuis la création, en 1996, du Progetto Amazzone², elle gère avec la journaliste Anna Barbera les Journées Biennales Internationales de Mythe, Science et Théâtre ainsi que le Teatro Studio Attrice / Non ${ }^{3}$, qui est l'atelier de recherche théâtrale du Centro Amazzone.

Après une longue production poétique, elle s'est consacrée à la dramaturgie: d'abord, à travers l'écriture de textes qui s'inscrivent dans la recherche de nouveaux langages en collaboration avec des metteurs en scène engagés; ensuite, par la réalisation de projets de mise en scène avec le Teatro Studio Attrice/Non, dont la production théâtrale s'inspire des grands personnages féminins de la tragédie et du mythe (Antigone, Cassandre, Penthésilée); elle collabore avec le Laboratorio di Drammaturgia Antica de l'Université de Pavie ${ }^{4}$. En 2003, elle écrit Lampedusa beach, choisie par le comité de lecture de la Comédie française qu'elle met en scène en 2011 toujours à la Comédie française, et en 2015 au Piccolo teatro de Milan. En 2015 elle reçoit le Prix de la critique théâtrale italienne.

\section{Le mythe entre crise économique et crise migratoire}

Si dans Lampedusa beach histoire contemporaine et mythe sont strictement liés dans un rapport dialectique où le récit mythique est un hypotexte moins apparent, dans des pièces comme Cassandra on the road et Programme Penthésilée la dimension mythique est évidente dès le début et les titres de ces pièces nous plongent au cœur du mythe tout en évoquant un contexte contemporain.

Rappelons que dans la tradition grecque la princesse troyenne Cassandre est liée au dieu Apollon qui, tombé amoureux de Cassandre, lui offre le don de prédire l'avenir. Cassandre refuse et Apollon se venge en décrétant que ses prédictions ne seront jamais crues. Par conséquent, Cassandre est isolée de sa communauté en raison du don qu'elle a reçu, un don qui la rend à la fois intangible et intouchable, incroyable et jamais crue. Elle est vouée à la tragédie de l'esseulement et son isolement est celui de la folie. Ajoutons aussi que dans le théâtre grec, outre la connaissance de l'avenir, au mythe de Cassandre est également associée la mémoire. C'est par exemple le cas de la tragédie d'Eschyle Agamemnon où, interpellée par le Coryphée à son arrivée à Argos, Cassandre montre qu'elle connaît les événements qui ont frappé la maison des Atrides sans pour autant les avoirs vécus:

2 http://www.progettoamazzone.it/.

3 http://www.progettoamazzone.it/teatro/attricenon/attricenon.aspx.

$4 \mathrm{http} / / /$ crimta.unipv.it/centro/il-centro. 
Rendez-moi témoignage: ai-je perdu la piste? Mon flair n'a-t-il pas éventé la trace des crimes commis jadis? C'est que cette maison loge une troupe bruyante qui jamais ne s'en éloigne [...] elle a bu du sang humain, et elle est à demeure sous ce toit dont on ne les chassera pas aisément: ce sont les Furies liées à la race. Elles assiègent les lieux, ces chanteuses, elles y chantent l'égarement qui fut la source et la racine de tout, en crachant l'une après l'autre leur indignation devant le lit incestueux qui a coûté cher à celui qui s'y vautrait (Eschyle 1999: 209).

À son tour, dans l'Iliade Penthésilée arrive à Troie avec douze autres Amazones, elle se distingue par ses nombreux exploits devant la ville assiégée puis succombe devant Achille (Homère 2014: III, 185-189). Celui-ci tombe amoureux d'elle en la voyant mourir. Au XIX siècle, Heinrich von Kleist recrée le mythe de Penthésilée dans sa tragédie éponyme en inversant les rôles: c'est la reine des amazones qui tue Achille et tombe amoureuse de lui.

Dans le théâtre de Lina Prosa tous ces personnages mythiques sont plongés dans la contemporanéité la plus proche: Cassandre prédit la crise économique aux USA, Penthésilée est confrontée au cancer du sein et Shaouba est l'une des victimes de la question de la crise migratoire.

Cassandre on the road ${ }^{5}$, écrite en 1998 et créée en 2003 au centre Amazzone de Palerme (2003, "Cassandra on the road apre Infiammazioni ») est sans doute l'une des pièces de Lina Prosa qui entretient le rapport le plus direct avec le mythe et notamment avec l'Iliade d'Homère et l'Agamemnon d'Eschyle. Ce rapport est immédiatement perceptible par le titre qui réunit la figure de la prophétesse grecque et le roman éponyme de Jack Kerouac en donnant ainsi immédiatement le cadre dans lequel se déroule l'intrigue.

Mais au-delà du titre et de la protagoniste qui a gardé la fonction prophétique de la princesse grecque de prévoir l'avenir sans être crue, l'histoire tragique de l'émigrée grecque est constamment croisée avec celle de la princesse Cassandre, fille du roi Priam et de la reine Hécube et sœur d'Hector.

Dans la pièce de Lina Prosa, Cassandre est une femme grecque émigrée aux USA. Elle est la fille d'un surveillant aux Marchés Généraux. Son passé mythique de prophétesse et de princesse troyenne est encore vivant et actif en elle, jusqu'à produire des souvenirs liés à l'Iliade et à la guerre de Troie. Dans le présent, Cassandre est licenciée par Coca-Cola parce qu'elle en a prédit la crise économique. Toujours refusée. Cassandre erre en nomade, continuant à ne pas être crue et traînant avec elle des morceaux de cannettes, des fils électriques, des résidus d'archéologie industrielle et de son passé mythique.

5 En France ce texte a fait l'objet de deux lectures: la première dirigée par Pascale Henry au festival Carta bianca à Chambéry, le 30 septembre 2007; la deuxième par Valérie Lang, dirigée par Christine Le Tailleur, à l'Odéon-Théâtre de l'Europe (Paris) le 25 novembre 2009, dans le cadre de Face à Face: paroles d'Italie pour les scènes de France. 
Le croisement entre le présent tragique de l'immigrée et le passé de la princesse grecque se produit dès le début du monologue, l'histoire contemporaine est croisée avec le mythe:

Je suis descendue du bateau en précédant les Grecs

auxquels il fallut un jour entier

pour décharger les cent caisses de pommes ramenées

de Grèce.

Le nouveau marché.

L'idée moderne de transformer

le mythe d'Hélène en un business (Prosa 2012: 49).

Ce mélange entre présent et passé mythique produit un effet de déclassement du mythe qui est confronté au monde contemporain, à un monde où les conditions de travail, très dégradées, sont proches de l'esclavage pour les travailleurs immigrés. Dans ce monde, l'usine a pris la place du palais des rois et est devenue le lieu de la tragédie contemporaine ${ }^{6}$. Le mythe est obligé de se conformer aux lois du marché et devenir un business.

Mon père, surveillant aux Marchés Généraux,

vécut de fluctuations.

Il fluctua dans son âme et dans son corps, comme

un pendule,

voyant croître, voyant baisser le prix des pommes (Prosa 2012: 50).

Victime des fluctuations des prix du marché, «l'Iliade des rois» se transforme en «l'Iliade des pauvres» et ses personnages sont emportés par la violence de la crise économique. Priam, le père, et Hector, le frère de Cassandre perdent leur vie à l'usine, tandis que Cassandre est licenciée et doit s'enfuir pour échapper à la mort (Prosa 2012: 50-51). La mort de son frère Hector est représentée «en contrechamp» avec la scène de sa fuite sur un char et les deux récits - le récit épique et celui de la fuite de l'usine - sont croisés et cristallisés à travers les mots de Cassandre:

Qui meurt sur un char véloce ou sur un pommier

ne mérite pas d'être dédommagé.

Qui meurt par désordre de nature

Ne pèse pas sur l'économie (Prosa 2012: 53).

\section{Un théâtre du fragment mythique}

Comme dans la tragédie grecque où l'auteur tirait de la tradition épique un fragment pour l'utiliser dans une autre combinaison d'événements capable de dire les universels (Sacco 2012: 17-18), dans Cassandre on the road Lina Prosa utilise de manière fragmentaire la narration épique. L'Iliade est constamment évoquée dans le monologue sous forme minimale soit pour évoquer le nom de

6 «Je suis dehors. / Les portes fermées de l'usine / comme si c'était le palais du roi», (Prosa 2012: 55). 
ses parents et de son frère, soit pour indiquer des objets - les trois pommes d'or, le bouclier d'Hector - dont la signification mythique suffit à éveiller l'image souhaitée dans la tête du spectateur.

Cependant, le fragment mythique ainsi utilisé n'acquiert pas une autonomie théâtrale. Il n'est pas recréé dans un ordre nouveau d'événements, mais il existe comme terme de confrontation avec la réalité historique de l'émigrée grecque pour souligner le déclassement de sa condition, ou bien pour affirmer l'identité du destin tragique de Cassandre dans la société capitaliste contemporaine.

À ce propos l'épisode du viol illustre bien ce rapport entre mythe et réalité contemporaine. Dans le fragment mythique utilisé que l'immigrée grecque raconte dans Cassandre on the road la princesse troyenne Cassandre est violée par le roi Agamemnon. Le viol est raconté d'une manière très réaliste comme ayant eu lieu pendant le voyage de retour à Mycènes. L'immigrée Cassandre précise même qu'au moment des faits ils se trouvaient à sept kilomètres de Mycènes et que l'agression s'est terminée par un accident lors duquel le char, sur lequel elle voyageait avec le roi Agamemnon, s'est renversé et brisé. Cependant, ni Homère ni Eschyle ne font référence à un tel épisode dans leurs œuvres. Le viol raconté par Cassandre s'est donc produit entre l'Iliade et l'Agamemnon c'est-à-dire après que le roi de Mycènes a obtenu la fille de Priam en esclave comme récompense de sa valeur dans la guerre de Troie et avant leur arrivée à Mycènes, qui constitue le début de la tragédie d'Eschyle.

En effet, ce qui est raconté au spectateur dans Cassandre on the road n'est pas le récit mythique tel qu'il a été transmis par les textes d'origine, mais le résultat d'une réécriture du mythe. En reprenant deux de ses principales sources, Lina Prosa procède à une recréation du fragment mythique pour l'utiliser dans une combinaison d'événements différents dont l'objectif est de dire l'horreur universelle provoquée par le viol, sujet récurrent dans le théâtre de Lina Prosa (Prosa 2012: 22-24). Toutefois, ce premier récit mythique ne semble pas suffire pour atteindre l'objectif qui est d'émouvoir. La narration de cette violence ne s'arrête pas là, car le premier récit est doublé d'un deuxième récit de viol qui marque également le passage du temps mythique au temps historique sans solution de continuité sur la scène. En effet, après le viol perpétré par le roi de Mycènes, Cassandre se retrouve dans un bar où elle subit une deuxième agression sexuelle par un Agamemnon prolétaire à l'allure monstrueuse: «un homme par hasard qui à la place de la main a une prothèse, un crochet de glace » (Prosa 2012: 64). Ainsi le récit de Cassandre fait-il glisser le spectateur de la recréation d'un fragment mythique à la tragédie contemporaine de l'émigrée grecque.

Le passage analysé montre bien que le récit de l'immigrée Cassandre possède une dimension ambiguë qui lui permet de passer aisément du mythe de la princesse troyenne à la réalité de l'immigrée grecque. Cette ambiguïté, comme l'explique la philosophe italienne Daniela Sacco dans son essai consacré aux rapports entre mythe et théâtre, est l'une des caractéristiques essentielles du 
mythe. La dimension mythique possède un caractère plurivoque dans sa capacité de dire les universels qui le conduit à maintenir dans l'ambivalence ce qui est univoque pour le logos, pour la pensée rationnelle (Sacco 2012: 64). L'ambiguïté permet la coexistence dans le théâtre de Lina Prosa d'un temps historique et d'un temps mythique. Le second permet une circularité entre passé et présent tandis que le temps historique est conçu uniquement en termes de progression chronologique.

La circularité de la dimension temporelle et sa perméabilité dans la linéarité du temps historique est une constante du théâtre de Lina Prosa où, comme nous l'avons vu, les fragments mythiques, de l'épopée et de la tragédie antique pénètrent l'histoire très contemporaine de l'émigrée grecque. Une circularité qui est renfoncée par des éléments linguistiques répétés constamment comme le «tel quel identique», véritable fil rouge linguistique de Cassandre on the road. La répétition de ces éléments linguistiques permet la coprésence du fragment mythique et du récit contemporain sans que le premier devienne métaphore du second. La coprésence dans le monologue d'éléments du mythe et d'éléments contemporains se transforme en confrontation dans un deuxième procédé circulaire qui à plusieurs moments met en contact une réplique de la princesse troyenne avec celle de l'immigrée grecque:

Moi, je te dis: pourquoi ne t'ont-ils pas crue?

Toi, tu me dis: répète: ne te croient-ils pas? (Prosa 2012: 55)

Le hic et nunc de la scène permet la coprésence d'un temps de l'histoire et d'un temps du mythe afin de produire une organisation qui n'est pas celle du monde «réel» du spectateur. Cependant, la représentation crée les conditions qui lui permettent de penser le monde dans lequel il vit (Biet-Triau 2006: 413-427). Autrement dit, dans le théâtre de Lina Prosa le rapport entre le temps historique et le temps mythique ne se pose pas en termes d'opposition, mais sous la forme d'un rapport dialectisé qui vise à produire une confrontation avec la signification de mythe survécue jusqu'à notre époque (Ubersfeld, 1996: 205-2011).

Nous retrouvons ces mêmes procédés d'écriture dramaturgique dans Lampedusa beach même si le récit mythique est moins directement présent que dans Cassandre on the road. Premier volet de la trilogie dite du naufrage avec Lampedusa snow (2012) et Lampedusa way (2014), Lampedusa beach est un long monologue de Shauba, une jeune femme africaine, après le naufrage du bateau de migrants clandestins qui aurait dû les faire débarquer à Lampedusa.

Comme Cassandre on the road, Lampedusa beach est inspiré d'un fait divers relaté par la presse en 2003 : le naufrage au large de l'île sicilienne de Lampedusa d'une charrette des mers qui transportait des centaines de migrants. L'auteur a voulu croiser ce fait divers avec le mythe d'Ulysse tel qu'Homère le raconte dans l'Odyssée et notamment au chant $\mathrm{V}$ où, à la suite de la tempête déchaînée par Poséidon, Ulysse fait naufrage et risque de perdre la vie avant d'être sauvé par la déesse Leucothée et de se retrouver sur l'île des Phéaciens. 
Parlant des conditions de création de Lampedusa beach, Lina Prosa a expliqué le recours aux mythes comme un processus inéluctable lié à sa culture d'origine: la culture sicilienne et méditerranéenne:

Dans Lampedusa beach, je transforme la réalité en mythe à travers la parole poétique. La parole poétique donne la possibilité à Shauba, la protagoniste de la pièce, de devenir mythique. Elle est mythique parce que son histoire parle à tout le monde. Elle peut parler au monde parce qu'elle n'est pas confinée dans l'espace limité d'une maison, d'une ville. C'est mon origine qui m'a conduite à une telle démarche. La tragédie grecque antique, celle d'Eschyle, de Sophocle, d'Euripide est pour moi ma nourriture théâtrale. Je dis souvent que nous n'avons pas qu'un âge chronologique, vingt ans, trente ans..., mais que nous avons 2500 ans. Nous venons de très très loin. C'est la littérature, c'est la parole poétique qui nous donne la possibilité de reconnaître cette mémoire?

Ces propos de l'auteure nous confirment encore une fois que le processus de mythification de la réalité est rendu possible à travers la parole poétique, ce qui permet au langage théâtral d'acquérir une identité forte et de se différencier du langage des médias. La mythification de la réalité contemporaine à travers la parole poétique permet à l'artiste, au dramaturge, de créer un personnage universel dont la parole, le discours est chargé de sens car il s'inscrit dans une mémoire mythique, une stratification mythique qui remonte à l'épopée et à la tragédie grecques.

Ainsi, Lampedusa beach nous renvoie au mythe d'Ulysse, à un mythe fondateur de la littérature et de la culture méditerranéennes qui autorise le recours à une construction cyclique avec des éléments qui rythment le monologue de Shauba: des objets auxquels on attribue une fonction poétique, une alternance entre discours et respiration, des sons porteurs d'une identité culturelle. Cette construction cyclique transforme la représentation en un rituel mythique où «les objets, les sons et les éléments de l'espace scénique, utilisés tels des images, des paroles, sont connectés entre eux selon les lois du symbolisme et des analogies vivantes» (Sacco 2012: 162).

Comme déjà dans Cassandre on the road, la parole de Lampedusa beach devient poétique aussi grâce à un traitement "symbolique» auquel sont soumis certains objets qui accompagnent Shauba dans sa traversée de la Méditerranée.

Les lunettes de soleil que Shauba a reçues en cadeau de sa tante Mahama ont une valeur magique et symbolique car elles lui permettent de regarder droit vers l'horizon, de contrôler l'arrivée et de rester à flot quelque temps: «Les lunettes opposent une résistance au poids du corps. C'est comme si elles voulaient me tirer vers le haut. Elles font comme chaloupe de sauvetage.» (Prosa 2012, circularité: 26)

7 "Lampedusa beach de Lina Prosa: le contexte menant à l'écriture», Théâtrecontemporain.net, http://www.theatre-contemporain.net/spectacles/LampedusaBeach-10372/videos/media/Lampedusa-Beach-de-Lina-Prosa-les-difficultes-decriture?autostart (consultée le 5 novembre 2016). Notre traduction. 
Le second objet symbolique - le bol de coco - est un objet clandestin car Shauba l'introduit en cachette sur le bateau. Mais il est également un objet anthropologique car il est le témoignage des conditions dramatiques de cette migration clandestine comme Shauba le dit dans son appel «au chef de l'État d'Affrique» :

Permettez-moi une dernière volonté... Si la marine militaire italienne récupère mon bol de coco, demandez au chef d'État italien de vous le rendre... Même si le dossier sera long n'y renoncez pas, rendez-le à Mahama... ou si vous voulez, mettez-le dans un musée... Je suis la représentante d'un phénomène social il faut en garder les traces... il en va de votre histoire monsieur le chef d'État, vous ne pouvez pas feindre qu'il n'y a rien. (Prosa 2012:35-36)

Ces deux objets, les lunettes et le bol de coco de Shaouba, s'opposent aux autres objets - faux passeports, sacs en plastiques, jeans élimés - qui flottent sur l'eau à côté des cadavres de migrants. Ces derniers ont perdu toute fonction pratique et poétique sauf celle d'être des signes de mort. (Prosa 2012:37-38)

Le deuxième procédé qui contribue à la construction de la langue poétique de Lampedusa beach est le traitement sonore appliqué au couple de mots: Afrique et Capitalisme qui, comme "tel quel identique» dans Cassandre on the road, constituent le fil rouge de la pièce.

Le $\mathrm{f}$ (spirant) du mot «Afrique» est multiplié une, deux, voire trois fois. Il apparaît en forme adjectivale dès le début de la pièce dans un échange de répliques entre Shauba et sa tante Mahama, dit par Shauba et il rappelle celui entre la princesse troyenne et l'immigrée grecque dans Cassandre on the road:

Je suis Mahama, l'Affricaine

Non, Mahama, tu es l'Africaine

Non, Shauba, je suis l'Affricaine

Non, Mahama, tu es l'Africaine

Non, Shauba, je suis l'Affricaine. (Prosa 2012: 11)

Le prolongement du son à l'intérieur du mot "Affrique» qui revient systématiquement dans le monologue de Shauba est une manière de mieux ressentir la saveur de sa terre ancestrale, de maintenir le dialogue avec Mahama et de garder cette mémoire des origines dont parle Lina Prosa à propos de son rapport à la culture méditerranéenne. (Prosa 2012: 14)

Le mot "CCappitalliste», qui subit le même traitement que le mot "Affrique» avec un dédoublement des consonnes, est évoqué en opposition au mot "Afrique». Mahama, qui possède la connaissance des choses, explique les conditions de pauvreté des Africains par le «Cappittallissmme». Dans le même temps, elle explique à sa nièce que la seule possibilité de survie pour Shauba est de rejoindre le monde des riches, «le monde des Ccappitalistes»:

Elle [Mahama] explique tout avec le Cappittallissmme,

Un jour on mange l'autre non.

Survivre, cela dépend de la bonté des CCappittallissttes. (Prosa 2012: 16)

Cette relation très étroite entre mythe, poésie et théâtre que nous avons soulignée dans l'œuvre théâtrale de Lina Prosa nous renvoie au théâtre de Pier 
Paolo Pasolini et notamment au lien direct que l'auteur d'Orgie établit entre la poésie et le théâtre. En 1966, lors d'une longue hospitalisation, Pier Paolo Pasolini décide d'écrire un théâtre inspiré de la tragédie grecque mais fortement ancré dans l'époque contemporaine. Un théâtre en vers, des vers très proches de la prose, mais qu'il voit comme une continuation du travail poétique commencé avec les Cendres de Gramsci:

(Dans mes poèmes en vers) moi, en vous parlant à la première personne, j'étais à la fois l'auteur et un véritable personnage d'un monologue. C'est cela qui m'a conduit au théâtre, d'une manière presque inéluctable. Ce n'est ni un mérite ni une faute, mais je crois que peu de poèmes sont conçus pour être lus «à haute voix» (pour être oralisés) comme le sont les miens. En effet, ils ne sont que rarement «objet»; ils sont presque toujours (et sans que moi je le veuille - comme un lettré ambitieux) suspendus, en cours de réalisation. Ce sont des confessions, des péroraisons, des méditations: en somme, des monologues, exactement comme ceux d'Hamlet ${ }^{8}$.

Cette réflexion de Pier Paolo Pasolini deviendra deux ans plus tard une véritable poétique théâtrale en opposition au théâtre traditionnel et au théâtre d'avant-garde avec la publication du manifeste pour un nouveau théâtre en 1968 ainsi que de plusieurs écrits qui précisent les caractéristiques de ce que Pasolini appelle «le théâtre de parole»:

Deux genres de théâtre sont désormais opérants: le théâtre traditionnel et le théâtre d'avant-garde, qui en réalité sont deux «formes différentes de théâtre». En tant qu'auteur dramatique, en écrivant mon premier texte, moi, j'ai choisi d'une manière intuitive une troisième voie: le théâtre de parole, comme je l'ai qualifié par la suite. Mais pourquoi de "parole»? Parce qu'il est écrit en vers, c'est-à-dire dans la «langue de la poésie»: dans ce théâtre, la parole est utilisée dans son moment d'expression maximale: à la fois «ambiguë» et «absolue».9

Cette nécessité d'absolu et cette plurivocité nous les retrouvons dans le théâtre de Lina Prosa. Dans Cassandre on the road et Lampedusa beach, le langage poétique joue un rôle de premier plan dans la réécriture du mythe sans pour autant que la question de la recréation du récit mythologique ne soit jamais une

8 "(Nelle poesie in versi) io, parlandovi in prima persona ero, insieme, l'autore e un vero e proprio personnaggio monologante. Ciò mi ha portato al teatro, quasi ineluttabilmente. Non è né un merito né un demerito, ma credo che poche poesie siano fatte per essere lette "ad alta voce» (a essere orali) come lo sono le mie. Esse infatti non sono che raramente "oggetto»; sono quasi sempre (e senza che io lo volessi - da letterato ambizioso) sospese, in lavorazione. Erano confessioni, perorazioni, meditazioni : insomma monologhi, proprio come quelli dell'Amleto...» (Naldini 1989: 302-303). Notre traduction.

9 «Operanti sono ormai due generi di teatro: il teatro tradizionale e il teatro d'avanguardia, che sono, in realtà due "teatri diversi". Io come autore teatrale, scrivendo il mio primo testo, ho preso, intuitivamente una terza via: il teatro di parola, come l'avrei poi definito. E perché mai di "parola"? Perché è scritto in versi, cioè nella "lingua della poesia": e in esso dunque "la parola" è usata in un suo momento espressivo al massimo grado: insieme "ambiguo" e "assoluto" (Pasolini 2001 : 347-351). Notre traduction. Voir aussi Pasolini 1999. 
question uniquement formelle. Dans son rapport dialectique avec une réalité très contemporaine comme celle de la crise migratoire, de la crise économique ou de la maladie, le mythe permet de rejouer, réagencer et confronter ce que le temps historique et humain sépare et dessine: une condition tragique absolument contemporaine.

Comme l'a souligné Jean-Paul Manganaro, dans un article sur le mythe dans le théâtre de Lina Prosa, la condition tragique des personnages de ce théâtre n'est pas l'opposition à une divinité, mais le contraste avec une situation politique. Cassandre, Penthésilée, Shaouba revendiquent en leur nom et en leur corps un combat contre la violence de ce qui se dessine comme une imposition humainemasculine, sociale, anthropologique. En un mot une imposition politique et non comme une fatalité du divin (Manganaro 2012: 115-125).

\section{Bibliographie}

Biet C. et Triau C., 2006, Qu'est-ce que le théâtre?, Paris, Gallimard.

"Cassandra on the road apre Infiammazioni», La Repubblica, 29 juin: http:// ricerca.repubblica.it/repubblica/archivio/repubblica/2003/06/29/cassandraon-the-road-apre-infiammazioni.html, consultée le 18 novembre 2015.

Eschyle, 1999, Agamemnon, in: Les Tragiques grecs (trad. V.-H. Debidour), Paris, Le livre de poche.

Homère, 2014, Iliade, (trad. P. Mazon), Paris, Les Belles Lettres.

Kleist H. (von), 1999, Penthésilée (trad. R. Orthmann et E. Recoing), Arles, Actes Sud.

Manganaro J.-P., 2912, «Lina Prosa: "Cassandre, Penthésilée, la Méditerranée. Nouveaux mythes, nouveaux chaos" ", in: Lina Prosa, Lampedusa beach, Besançon, Les Solitaires Intempestifs, p. 115-125.

Naldini N., 1989, Pasolini, una vita, Turin, Einaudi.

Pasolini P.P., 2001, Teatro, W. Siti et S. De Laude (dir.), Milan, Meridiani Mondadori.

Pasolini P.P., [1968] 1999, «Manifesto per un nuovo teatro», in: P. P. Pasolini, Teatro, Milan, Garzanti.

Prosa L., 2012, Cassandre on the road, (trad. J.-P. Manganaro), Besançon, Les Solitaires intempestifs.

Prosa L., 2012, Lampedusa beach, (trad. J.-P. Manganaro), Besançon, Les Solitaires Intempestifs.

Sacco D., 2012, Mito e teatro. Il principio drammaturgico del teatro, Milan, Udine, Mimesis Edizioni.

Ubersfeld A., 1996, Lire le théâtre II. L'école du spectateur, Paris, Éditions Belin. 